\title{
EXPANSIONS OF ANALYTIC FUNCTIONS
}

\author{
BY \\ R. P. BOAS, JR.
}

Introduction. There is an extensive literature dealing with the problem of expanding analytic functions of a complex variable in generalized Taylor series of the form

$$
f(z)=\sum_{n=0}^{\infty} c_{n} g_{n}(z)
$$

where the $g_{n}(z)$ are, in a suitable sense, "nearly" the functions $z^{n}\left({ }^{1}\right)$. If $g_{n}(z)=z^{n}\left[1+h_{n}(z)\right]$, where the $h_{n}(z)$ are analytic and bounded in a circle $|z|<r$ and vanish at $z=0$, and $f(z)$ is analytic in $|z|<r$, the possibility of an expansion of the form (1) was established by S. Pincherle [9]; the series converges to $f(z)$ in some circle $|z|<s$, where in general $s<r$. Much of the later work has been devoted to obtaining better estimates for the number $s$. In this paper, a new attack on the problem is developed; it eliminates rearrangements of power series, and uses a criterion for "nearness" of two sequences of functions which is essentially contained in work of Paley and Wiener [26, p. 100] (where it is applied to another problem). The results include some of those of G. S. Ketchum [4], which are the most precise yet obtained, and in part go beyond them. Well known expansion theorems of G. D. Birkhoff [1] and J. L. Walsh [17] are also obtained.

The simplest of my results (and the most convenient one for applications) is that if the functions $g_{n}(z)$ in (1) are of the form specified above, and if the $h_{n}(z)$ have a common majorant $h(z)$ for large $n$ (that is, if the coefficients in the power series of $h_{n}(z)$ are less in absolute value than the corresponding coefficients of $h(z)$ ), then the expansion (1) converges to $f(z)$ in $|z|<s$ if $h(s)<1$. For example, if $1+h_{n}(z)=e^{\alpha_{n} z}$, with $\lim \sup _{n \rightarrow \infty}\left|\alpha_{n}\right| \leqq 1$, we may take $h(z)=e^{(1+\epsilon) z}-1$ (with any positive $\epsilon$ ), so that the region of convergence of (1) is at least $|z|<\log 2$; I have not been able to establish convergence in a larger region than $|z|<1 / e$ by using the theorems in the literature $\left({ }^{2}\right)$.

It is also possible to restrict linear combinations of the coefficients of the

Presented to the Society, April 27, 1940; received by the editors March 18, 1940.

(1) The bibliography at the end of this paper contains all the references which $I$ have found (without however making an intensive search of the literature) on general expansions of this type. For special theorems, other than those considered in this paper, see especially G. S. Ketchum [4]. (Numbers in brackets refer to the bibliography.)

(2) Added in proof: Ibragimoff [32] has proved that every function analytic in $|z|<s$ is the uniform limit in $|z| \leqq s^{\prime}<s$ of a sequence of linear combinations of the functions in question if $s \leqq \log 2$. 
$h_{n}(z)$ instead of the coefficients themselves; this can be done by a method different from that used by G. S. Ketchum in obtaining the first such results (see \$5). Another generalization consists in modifying the assumption that the functions $g_{n}(z)$ should have precisely the form $z^{n}\left[1+h_{n}(z)\right]$ (see Theorem 6.4).

The expansion theorems of this paper were originally developed in the hope (which has so far proved illusory) of settling a conjecture concerning the values taken by derivatives of entire functions. However, I have obtained some new results in this field. In particular, I prove the following theorem (Theorem 7.1): If $f(z)$ is an entire function of exponential type $k<\log 2$, with $f(0)=1$, and if the points $\alpha_{n}(n=0,1,2, \cdots)$ are in the circle $|z| \leqq 1$, then for every $r<k$

$$
\sum_{n=0}^{\infty} \frac{\left|f^{(n)}\left(\alpha_{n}\right)\right|^{2}}{r^{2 n}} \geqq 2 e^{r}-e^{2 r} .
$$

This generalizes a theorem of S. Takenaka $\left(^{(3}\right)$ which states that $f^{(n)}\left(\alpha_{n}\right)$ cannot be zero for all $n$.

Many of the papers listed in the bibliography treat, besides the convergence of the series (1), the existence of systems of functions biorthogonal to the $g_{n}(z)$, the form of the coefficients in (1), etc. These problems are not considered in this paper, although its methods could be made to furnish information about them.

Some of the results of this paper were announced, with indications of the proofs, in a note in the Proceedings of the National Academy of Sciences $\left({ }^{4}\right)$.

1. Abstract expansion theorems. We consider a normed complex linear space $E$, and a sequence $G=\left\{x_{n}\right\}$ of elements of $E$. $G$ is said to be a fundamental set if the set of all finite linear combinations of elements of $G$ is everywhere dense in $E$; that is, if for every $y \varepsilon E$ there exist complex numbers $c_{k, n}$ such that

$$
y=\lim _{n \rightarrow \infty} \sum_{k=1}^{\infty} c_{k, n} x_{k} .
$$

$G$ is said to be a base if every element $y \varepsilon E$ has a unique representation as an infinite series of multiples of elements of $E$; that is, if for every $y \varepsilon E$ there exists a unique sequence of complex numbers $c_{k}$ such that

$$
y=\lim _{n \rightarrow \infty} \sum_{k=1}^{n} c_{k} x_{k}
$$

The following theorem states in effect that a sequence sufficiently near

(3) See J. M. Whittaker [30, p. 44]; Takenaka [29].

(4) Vol. 26 (1940), pp. 139-143. 
another sequence which is a fundamental sequence or a base is also a fundamental sequence or a base.

TheOREM 1.1. Let the sequences $G=\left\{x_{n}\right\}$ and $H=\left\{y_{n}\right\}$ have the property that for some number $\lambda(0<\lambda<1)$, and for all finite sequences $a_{1}, a_{2}, \cdots, a_{N}$ of complex numbers,

$$
\left\|\sum_{n=1}^{N} a_{n}\left(x_{n}-y_{n}\right)\right\| \leqq \lambda\left\|\sum_{n=1}^{N} a_{n} x_{n}\right\| .
$$

Then

(i) if $G$ is a fundamental set, so is $H$;

(ii) if $E$ is complete and $G$ is a base, $H$ is a base.

In case (ii), furthermore, if the element $x \varepsilon E$ has the expansion

$$
\sum_{k=1}^{\infty} c_{k} y_{k}
$$

the coefficients $c_{k}$ have the property

$$
\left\|\sum_{k=1}^{\infty} c_{k} x_{k}\right\| \leqq \frac{1}{1-\lambda}\|x\|
$$

Theorem 1.1 (ii), in the special form which it assumes when $G$ is a normal orthogonal base, was given (with a proof which applies to a general base $G$ ) by Paley and Wiener $\left[26\right.$, p. 100] for the Hilbert space $L^{2}(-\pi, \pi)$. For a general Banach space, the proof given by Paley and Wiener needs only formal modifications; in this paper, Theorem 1.1 (ii) will be used almost exclusively for Hilbert spaces, and is consequently established by the proof of Paley and Wiener (since all realizations of abstract Hilbert space are equivalent). We omit the proof of Theorem 1.1 (ii).

The proof of Theorem 1.1 (i) is considerably simpler; this part would be sufficient for the applications which will be made in $\$ 7$ to derivatives of analytic functions. We suppose that $G$ is fundamental, that $H$ is not, and that (1.3) is satisfied. Then there is a linear $\left(^{(5)}\right.$ functional $f$, defined on $E$, such that $f\left(y_{n}\right)=0, n=1,2, \cdots$, while $f(z) \neq 0$ for some $z$. Let

$$
f\left(x_{n}\right)=f\left(x_{n}-y_{n}\right)=c_{n} \quad(n=1,2, \cdots) .
$$

Let $M=\|f\|$; that is, let $M$ be the smallest number such that, for all $x \varepsilon E$, $|f(x)| \leqq M\|x\|$. Then for any sequence $\left\{a_{n}\right\}$

$$
\left|\sum_{n=1}^{N} a_{n} c_{n}\right| \leqq M\left\|\sum_{n=1}^{N} a_{n}\left(x_{n}-y_{n}\right)\right\| \leqq M \lambda\left\|\sum_{n=1}^{N} a_{n} x_{n}\right\| .
$$

(5) "Linear" means "distributive and continuous," as in Banach's book [21]. 
Hence $\left(^{6}\right)$ there is a linear functional $g$, defined on $E$, such that $g\left(x_{n}\right)=c_{n}$ $(n=1,2, \cdots)$, and $\|g\| \leqq M \lambda<M$. But since $\left\{x_{n}\right\}$ is a fundamental set and $f\left(x_{n}\right)-g\left(x_{n}\right)=0(n=1,2, \cdots)$, we must have $f(x)=g(x)$ for every $x$, and consequently $M=\|f\|=\|g\| \leqq \lambda M<M$, a contradiction; for $M$ is not zero because $f(z) \neq 0$ for some $z$.

2. General expansions of analytic functions. We now apply Theorem 1.1 to the spaces $H_{p}(r)$ whose elements are functions $f(z)$ analytic in $|z|<r$, belonging to $L^{p}(p \geqq 1)$ in this circle; that is, each function $f(z)$ is assumed to satisfy $\left({ }^{7}\right)$

$$
\left\{\frac{1}{2 \pi} \int_{0}^{2 \pi}\left|f\left(\rho e^{i \theta}\right)\right| p d \theta\right\}^{1 / p} \leqq A, \quad 0 \leqq \rho<r,
$$

where $A$ depends only on $f$. It is well known $\left(^{8}\right)$ that if $f(z)$ satisfies (2.1) it has boundary values almost everywhere on $|z|=r$, and that the boundary function belongs to $L^{p}$. We complete the definition of $H_{p}(r)$ by defining the norm of $f(z)$ by the relation

$$
\|f\|=\left\{\frac{1}{2 \pi} \int_{0}^{2 \pi}\left|f\left(r e^{i \theta}\right)\right| p d \theta\right\}^{1 / p}
$$

We introduce, to save repetition, the following

Definition. $A$ sequence $\left\{f_{n_{i}}(z)\right\}$ of functions analytic in $|z|<r$ and belonging to some class $H_{p}(r)(1 \leqq p \leqq \infty)$ has Property $\mathrm{T}$ in $|z|<r$ if every function $f(z)$ analytic in $|z|<r$ and continuous in $|z| \leqq r$ can be expanded in a unique series of the form

$$
f(z)=\sum_{n=1}^{\infty} c_{n} f_{n}(z)
$$

the series converging uniformly in every circle $|z| \leqq r^{\prime}<r$. If furthermore the series in (2.2) converges uniformly in $|z| \leqq r$, the sequence has Property $\mathrm{T}_{\infty}$.

The sequence $\left(1, z, z^{2}, \cdots\right)$ is an obvious example of a sequence having Property $\mathrm{T}_{\infty}$ in any circle.

Applied to the spaces $H_{p}(r)$, Theorem 1.1 yields

THEOREM 2.1. Let $\left\{f_{n}(z)\right\}$ and $\left\{g_{n}(z)\right\}$ be two sequences of elements of $H_{p}(r)$, such that for some numbers $p$ and $\lambda(1 \leqq p \leqq \infty, 0<\lambda<1)$, and for all sets of complex numbers $a_{1}, a_{2}, \cdots, a_{N}$

(6) Banach [21, p. 56]. The result remains valid for complex linear spaces: see Bohnenblust and Sobczyk [23].

${ }^{(7)}$ Expressions involving $p$ are to be interpreted according to the usual conventions when $p=\infty$ : that is, as the limits as $p \rightarrow \infty$ of the corresponding expressions for finite $p$.

(8) See, e.g., Zygmund [31, p. 162]. 


$$
\begin{aligned}
\left\{\int_{0}^{2 \pi}\left|\cdot \sum_{n=1}^{N} a_{n}\left[f_{n}\left(r e^{i \theta}\right)-g_{n}\left(r e^{i \theta}\right)\right]\right|^{p} d \theta\right\}^{1 / p} & \\
& \leqq \lambda\left\{\int_{0}^{2 \pi}\left|\sum_{n=1}^{N} a_{n} f_{n}\left(r e^{i \theta}\right)\right|^{p} d \theta\right\}^{1 / p} .
\end{aligned}
$$

Then, in $|z|<r,\left\{g_{n}\right\}$ has Property $\mathrm{T}$ if $\left\{f_{n}\right\}$ has Property $\mathrm{T}$; if (2.3) is satisfied with $p=\infty,\left\{g_{n}\right\}$ has Property $\mathrm{T}_{\infty}$ if $\left\{f_{n}\right\}$ has Property $\mathrm{T}_{\infty}$. Moreover, if the expansion of $f(z)$ in terms of $\left\{g_{n}(z)\right\}$ has the form

$$
f(z)=\sum_{n=1}^{\infty} c_{n} g_{n}(z)
$$

the coefficients $c_{n}$ have the property

$$
\left\{\int_{0}^{2 \pi}\left|\sum_{k=1}^{\infty} c_{k} f_{k}\left(r e^{i \theta}\right)\right|^{p} d \theta\right\}^{1 / p} \leqq \frac{1}{1-\lambda}\left\{\int_{0}^{2 \pi}\left|f\left(r e^{i \theta}\right)\right| p d \theta\right\}^{1 / p} .
$$

The direct deduction from Theorem 1.1 is that the series in (2.4) converges to $f(z)$ in the topology of $H_{p}(r)$. In case $p=\infty$, this is the desired conclusion. Otherwise, if $|z| \leqq s<r$ we have

$$
\left|f(z)-\sum_{n=1}^{N} c_{n} g_{n}(z)\right|=\left|\frac{1}{2 \pi i} \int_{|w|=r}\left\{f(w)-\sum_{n=1}^{N} c_{n} g_{n}(w)\right\} \frac{d w}{w-z}\right| ;
$$

an application of Hölder's inequality shows that

$$
\lim _{N \rightarrow \infty}\left|f(z)-\sum_{n=1}^{N} c_{n} g_{n}(z)\right|=0,
$$

uniformly in $|z| \leqq s$.

We shall use Theorem 2.1 most frequently in the special case when $f_{n}(z)=z^{n-1}$. It then becomes

Theorem 2.2. The sequence $\left\{g_{n}(z)\right\}$ has Property $\mathrm{T}$ in $|z|<r$ if, for all sets of complex numbers $a_{0}, a_{1}, \cdots, a_{N}$,

$$
\begin{aligned}
\left\{\int_{0}^{2 \pi}\left|\sum_{n=0}^{N} a_{n}\left[r^{n} e^{i n \theta}-g_{n}\left(r e^{i \theta}\right)\right]\right|^{p} d \theta\right\}^{1 / p} & \\
& \leqq \lambda\left\{\int_{0}^{2 \pi}\left|\sum_{n=0}^{N} a_{n} r^{n} e^{i n \theta}\right|^{p} d \theta\right\}^{1 / p},
\end{aligned}
$$

where $p$ and $\lambda$ satisfy $1 \leqq p \leqq \infty, 0<\lambda<1$. If (2.6) is true with $p=\infty$, the sequence has Property $\mathrm{T}_{\infty}$.

From Theorem 2.2 we can deduce in a few lines the following generalization of expansion theorems of G. D. Birkhoff [1] and J. L. Walsh [17]. 
THEOREM 2.3. If the functions $g_{n}(z)$ are analytic in $|z|<r$, continuous in $|z| \leqq r$, and satisfy

$$
\sum_{n=0}^{\infty} r^{-2 n}\left|g_{n}(z)-z^{n}\right|^{2}<1
$$$$
|z|=r,
$$

the series converging uniformly, then the set $\left\{g_{n}(z)\right\}$ has Property $\mathrm{T}_{\infty}$ in every circle $|z|<s \leqq r$.

In Birkhoff's theorem, (2.7) is replaced by

$$
\sum_{n=0}^{\infty} r^{-n}\left|g_{n}(z)-z^{n}\right|<1,
$$$$
|z|=r
$$

this condition implies (2.7), by Cauchy's inequality. In Walsh's theorem (2.7) holds, and in addition the series in (2.8) is assumed to converge.

We apply the case $p=. \infty$ of Theorem 2.2. The sum of the series in (2.7) is continuous on $|z|=s$, when $s \leqq r$, and so has a maximum $\lambda^{2}<1$. We have, with $z=e^{i \theta}$,

$$
\begin{aligned}
& \max _{0 \leqq \theta \leqq 2 \pi}\left|\sum_{n=0}^{N} a_{n}\left[g_{n}(z)-z^{n}\right]\right| \\
& \leqq\left(\sum_{n=0}^{N}\left|a_{n}\right|{ }^{2} s^{2 n}\right)^{1 / 2} \max _{0 \leqq \theta \leqq 2 \pi}\left(\sum_{n=0}^{N} s^{-2 n}\left|g_{n}(z)-z^{n}\right|^{2}\right)^{1 / 2} \\
& \leqq \lambda\left(\frac{1}{2 \pi} \int_{0}^{2 \pi}\left|\sum_{n=0}^{N} a_{n} z^{n}\right|{ }^{2} d \theta\right)^{1 / 2} \\
& \leqq \lambda \max _{0 \leqq \theta \leqq 2 \pi}\left|\sum_{n=0}^{N} a_{n} z^{n}\right| .
\end{aligned}
$$

This establishes (2.6) with $p=\infty$, and Theorem 2.3 follows.

In this section we have applied part (ii) of Theorem 1.1. The weaker part (i) would yield a weak form of Property $T$ with the uniformly convergent series replaced by a uniformly convergent sequence of linear combinations.

3. Criteria for the existence of expansion theorems. From now on, we shall use Theorem 2.2 exclusively in the case $p=2$, which is the case in which criteria for the validity of (2.6) are most easily set up. Our functions $g_{n}(z)$ will, in this section, be of the form

$$
g_{n}(z)=z^{n}\left[1+h_{n}(z)\right] \quad(n=0,1,2, \cdots),
$$

where

$$
h_{n}(z)=\sum_{k=1}^{\infty} \gamma_{k}^{(n)} z^{k} \quad\left(|z|<r_{0}\right)
$$


We assume to begin with that the $h_{n}(z)$ have a common majorant $h(z)$; that is, that

$$
\left|\gamma_{k}^{(n)}\right| \leqq \delta_{k} \quad(k=1,2, \cdots ; n=0,1,2, \cdots),
$$

where $h(z)=\sum_{k=1}^{\infty} \delta_{k} z^{k}\left(|z|<r_{0}\right)$. This restriction will be considerably relaxed in $\$ 4$. We introduce the quantity $K_{\rho}$ by the definition

$$
K_{\rho}^{2}=\frac{1}{2 \pi} \int_{0}^{2 \pi}\left|h\left(\rho e^{i \theta}\right)\right|^{2} d \theta=\sum_{k=1}^{\infty} \delta_{k \rho}^{2} 2 k \quad\left(\rho<r_{0}\right) .
$$

TheOREM 3.1. The functions $g_{n}(z)$ have Property $\mathrm{T}$ in any circle $|z|<s$ provided that one of the following conditions is satisfied:

$$
\begin{gathered}
h(s)<1, \\
s<\sup _{0 \leqq \rho<r_{0}} \frac{\rho}{\left(K_{\rho}^{2}+1\right)^{1 / 2}} .
\end{gathered}
$$

We have to verify (2.6) with $p=2, r=s$, for an arbitrary set $\left(a_{0}, a_{1}, \cdots, a_{N}\right)$. We write

$$
\begin{gathered}
a_{n}^{\prime}= \begin{cases}a_{n}, & n=0,1, \cdots, N, \\
0, & n>N ;\end{cases} \\
\psi(z)=\sum_{n=0}^{\infty}\left|a_{n}^{\prime}\right| z^{n}, \quad \psi_{m}(z)=\sum_{n=0}^{m}\left|a_{n}^{\prime}\right| z^{n} .
\end{gathered}
$$

Then condition (2.6) takes the form

$$
\begin{aligned}
\Phi(r) I & \equiv \frac{1}{2 \pi} \int_{0}^{2 \pi}\left|\sum_{n=0}^{\infty} a_{n}^{\prime} r^{n} e^{i n \theta} h_{n}\left(r e^{i \theta}\right)\right|^{2} d \theta \\
& \leqq \lambda^{2} \sum_{n=0}^{\infty}\left|a_{n}^{\prime}\right|{ }^{2} r^{2 n} .
\end{aligned}
$$

$\Phi(r)$ can be rewritten as follows.

$$
\begin{aligned}
\Phi(r) & =\frac{1}{2 \pi} \int_{0}^{2 \pi}\left|\sum_{n=0}^{\infty} a_{n}^{\prime} r^{n} e^{i n \theta} \sum_{k=1}^{\infty} \gamma_{k}^{(n)} r^{k} e^{i k \theta}\right|^{2} d \theta \\
& =\frac{1}{2 \pi} \int_{0}^{2 \pi}\left|\sum_{m=1}^{\infty} r^{m} e^{i m \theta} \sum_{n=0}^{m-1} a_{n}^{\prime} \gamma_{m-n}^{(n)}\right|^{2} d \theta \\
& =\sum_{m=1}^{\infty} r^{2 m}\left|\sum_{n=0}^{m-1} a_{n}^{\prime} \gamma_{m-n}^{(n)}\right|^{2} .
\end{aligned}
$$

In the first place, we evidently have 


$$
\Phi(r) \leqq \sum_{m=1}^{\infty} r^{2 m}\left\{\sum_{n=0}^{m-1}\left|a_{n}^{\prime}\right| \delta_{m-n}\right\}^{2} \text {. }
$$

If we retrace the steps in (3.7), we then find

$$
\begin{aligned}
\Phi(r) & \leqq \frac{1}{2 \pi} \int_{0}^{2 \pi}\left|\sum_{n=0}^{\infty}\right| a_{n}^{\prime}\left|r^{n} e^{i n \theta} \sum_{k=1}^{\infty} \delta_{k} r^{k} e^{i k \theta}\right|^{2} d \theta \\
& =\frac{1}{2 \pi} \int_{0}^{2 \pi}\left|h\left(r e^{i \theta}\right) \psi\left(r e^{i \theta}\right)\right|^{2} d \theta \\
& \leqq\{h(r)\}^{2} \frac{1}{2 \pi} \int_{0}^{2 \pi}\left|\psi\left(r e^{i \theta}\right)\right|^{2} d \theta \\
& =\{h(r)\}^{2} \sum_{n=0}^{\infty}\left|a_{n}^{\prime}\right|^{2} r^{2 n} .
\end{aligned}
$$

Thus if $h(r)<1,(3.6)$ is satisfied; this proves Theorem 3.1 under condition (3.4).

We now observe that the expression

$$
\sum_{n=0}^{m-1}\left|a_{n}^{\prime}\right| \delta_{m-n}
$$

which occurs on the right of (3.8) is the coefficient of $z^{m}$ in the power series of $\psi_{m-1}(z) h(z)$, and consequently can be written as

$$
\frac{1}{2 \pi i} \int_{\mid z i=\rho} \frac{h(z) \psi_{m-1}(z)}{z^{m+1}} d z \quad\left(|z|=\rho<r_{0}\right) .
$$

Hence its square does not exceed

$$
\frac{1}{\rho^{2 m}} \frac{1}{2 \pi} \int_{0}^{2 \pi}\left|h\left(\rho e^{i \theta}\right)\right|^{2} d \theta \frac{1}{2 \pi} \int_{0}^{2 \pi}\left|\psi_{m-1}\left(\rho e^{i \theta}\right)\right|^{2} d \theta=\frac{K_{\rho}^{2}}{\rho^{2 m}} \sum_{n=0}^{m-1}\left|a_{n}{ }^{\prime}\right|^{2} \rho^{2 n}
$$

From (3.8) we now obtain, if $r<\rho$,

$$
\begin{aligned}
\Phi(r) & \leqq K_{\rho}^{2} \sum_{m=1}^{\infty}\left(\frac{r}{\rho}\right)^{2 m} \sum_{n=0}^{m-1}\left|a_{n}^{\prime}\right|^{2} \rho^{2 n} \\
& =K_{\rho}^{2} \sum_{n=0}^{\infty}\left|a_{n}^{\prime}\right|{ }^{2} r^{2 n} \sum_{m=1}^{\infty}\left(\frac{r}{\rho}\right)^{2 m}=\cdot K_{\rho}^{2} \frac{r^{2}}{\rho^{2}-r^{2}} \sum_{n=0}^{\infty}\left|a_{n}{ }^{\prime}\right|{ }^{2} r^{2 n} .
\end{aligned}
$$

Then (3.6) is satisfied if we can choose $\rho$ so that $K_{\rho}^{2} r^{2} /\left(\rho^{2}-r^{2}\right)<1$, or so that

$$
r^{2}<\frac{\rho^{2}}{K_{\rho}^{2}+1}
$$


This shows that the relation (3.6) is satisfied if $r=s$ and $s$ satisfies inequality (3.5).

In earlier theorems of the same type as Theorem 3.1, the conditions have restricted the coefficients $\delta_{k}$ of the function $h(z)$ majorizing the $h_{n}(z)$; here we restrict only the behavior of $h(z)$ in the large $\left({ }^{9}\right)$. Two less precise known theorems can be deduced as corollaries of Theorem 3.1.

TheOREM 3.2(10). If $\left|h_{n}(z)\right| \leqq M(\rho)(n=0,1,2, \cdots ;|z| \leqq \rho)$, then the functions $g_{n}(z)$ have Property $\mathrm{T}$ in $|z|<s$ if

$$
s<\sup _{0 \leqq \rho<r_{0}} \frac{\rho}{M(\rho)+1} .
$$

In fact, Cauchy's inequalities for derivatives yield, for $0<\rho<r_{0}$,

$$
\left|\gamma_{k}^{(n)}\right| \leqq M(\rho) \rho^{-k} \quad(n=0,1,2, \cdots ; k=1,2, \cdots)
$$

Consequently, if $\rho<r_{0}$, we can take $\delta_{k}=M(\rho) \rho^{-k}(k=1,2, \cdots)$; we then have $h(r)=r M(\rho) /(\rho-r)$, and $h(r)<1$ if $r<\rho /[M(\rho)+1]$. If we choose $\rho$ in the most favorable way, Theorem 3.2 follows from Theorem 3.1.

THEOREM $3.3\left({ }^{11}\right)$. If

$$
L_{\rho}=\sup _{1 \leqq k<\infty} \delta_{k} \rho^{k} \quad\left(\rho<r_{0}\right),
$$

the functions $g_{n}(z)$ have Property $\mathrm{T}$ in $|z|<s$ if

$$
s<\sup _{0 \leqq \rho<r_{0}} \frac{\rho}{L_{\rho}+1} .
$$

We have

$$
\begin{aligned}
\delta_{k} & \leqq \rho^{-k} L_{\rho} ; \\
h(r) & \leqq L_{\rho} \sum_{n=1}^{\infty}\left(\frac{r}{\rho}\right)^{n} \\
& =L_{\rho} \frac{r}{\rho-r} ;
\end{aligned}
$$

hence $h(s)<1$ if $s$ satisfies (3.11). The conclusion follows from Theorem 3.1.

For use in $\$ 5$, we note the following property of the coefficients in the expansion whose existence is established by Theorems 3.1 and 3.2. If $f(z)$ has the expansion

( $\left.{ }^{9}\right)$ However, Theorem 3.1 (even as generalized in $\$ 4$ ) does not seem to include Theorem III of G. S. Ketchum [4].

(10) Narumi [7], Takenaka [15], G. S. Ketchum [4].

(11) Graesser [2]. See also G. S. Ketchum [4, p. 215, footnote]. 


$$
f(z)=\sum_{n=0}^{\infty} c_{n} g_{n}(z)
$$

there is a number $A(s)$, not depending on $f(z)$, such that

$$
\sum_{n=0}^{\infty}\left|c_{n}\right|^{2} s^{2 n} \leqq A(s) \int_{0}^{2 \pi}\left|f\left(s e^{i \theta}\right)\right|^{2} d \theta
$$

This follows from the last part of Theorem 2.1.

4. Improvement of the criteria. The conditions established in $\$ 3$ can be generalized by restricting the $h_{n}(z)$ only for large $n$. The generalized conditions occur in part in the literature, and in part are new. It turns out that only the behavior of the $h_{n}(z)$ for large $n$ is relevant to the existence of expansions of the type which we consider, as the following lemma shows.

Lemma. Suppose that $g_{n}(z)$ and $g_{n}^{*}(z)$ are analytic in $|z|<r_{0}$, and $g_{n}(z) \equiv g_{n}^{*}(z)$ for $n>N$. If $\left\{g_{n}(z)\right\}$ has Property $\mathrm{T}$ in $|z|<s_{1}<r_{0}$, and $\left\{g_{n}^{*}(z)\right\}$ has Property $\mathrm{T}$ in every circle $|z|<s^{*} \leqq s_{2}$, where $r_{0} \geqq s_{2}>s_{1}$, then $\left\{g_{n}(z)\right\}$ has Property $\mathrm{T}$ in $|z|<s_{2}$.

Let $F(z)$ be an arbitrary function analytic in $|z|<s_{2}$ and continuous in $|z| \leqq s_{2}$, and let

$$
F(z)=\sum_{n=0}^{\infty} c_{n} g_{n}(z)
$$

where the series converges uniformly in any circle $|z| \leqq s_{1}^{\prime}<s_{1}$. Define a function $G(z)$ by the relation

$$
G(z)=F(z)-\sum_{n=0}^{N} c_{n} g_{n}(z)=\sum_{n=N+1}^{\infty} c_{n} g_{n}(z)=\sum_{n=N+1}^{\infty} c_{n} g_{n}^{*}(z) .
$$

Now $G(z)$ has a unique expansion of the form

$$
G(z)=\sum_{n=0}^{\infty} d_{n} g_{n}^{*}(z),
$$

the series converging uniformly in $|z| \leqq s_{2}^{\prime}<s_{2}$. By comparison with (4.2), we see that $d_{n}=0(n=0,1,2, \cdots, N)$. Hence the series in (4.1) converges uniformly in $|z| \leqq s_{2}^{\prime}$, and necessarily converges to $F(z)$. Since $s_{2}^{\prime}$ is any number less than $s_{2}$, the proof is complete.

We now suppose, as in $\S 3$, that $g_{n}(z)=z^{n}\left[1+h_{n}(z)\right]$, where

$$
h_{n}(z)=\sum_{k=1}^{\infty} \gamma_{k}^{(n)} z^{k} \quad(n=0,1,2, \cdots) .
$$

We suppose further that 


$$
\left|\gamma_{k}^{(n)}\right| \leqq \delta_{k}^{(n)} \quad(k=1,2, \cdots ; n=0,1,2, \cdots) .
$$

where the series

$$
H_{n}(z)=\sum_{k=1}^{\infty} \delta_{k}^{(n)} z^{k} \quad(n=0,1,2, \cdots)
$$

converge in $|z|<r_{0}$. We introduce, for $\rho<r_{0}$, the quantities

and we then set

$$
K_{\rho, n}^{2}=\sum_{k=1}^{\infty}\left(\delta_{k}^{(n)} \rho^{k}\right)^{2}, \quad L_{\rho}^{(n)}=\sup _{1 \leqq k<\infty} \delta_{k}^{(n)} \rho^{k}
$$

$$
K_{\rho}=\limsup _{n \rightarrow \infty} K_{\rho, n}, \quad L_{\rho}=\limsup _{n \rightarrow \infty} L_{\rho}^{(n)}, \quad h(r)=\limsup _{n \rightarrow \infty} H_{n}(r)
$$

$$
\left(0<r<r_{0}\right) \text {. }
$$

Then we can state

THEOREM 4.1. The functions $g_{n}(z)$ have Property $\mathrm{T}$ in any circle $|z|<s$ provided that $s$ satisfies one of the following three conditions $\left({ }^{12}\right)$ :

$$
\begin{aligned}
h(s) & <1, \\
s & <\sup _{0 \leqq \rho<r_{0}} \frac{\rho}{\left(K_{\rho}^{2}+1\right)^{1 / 2}}, \\
s & <\sup _{0 \leqq \rho<r o} \frac{\rho}{L_{\rho}+1} .
\end{aligned}
$$

Theorem 4.1 states that Theorems 3.1 and 3.3 remain valid when $K_{\rho}, L_{\rho}$, and $h(r)$ are defined by (4.4). The theorem follows at once from the lemma, with $g_{n}^{*}(z)=z^{n}$ for $n<N$, where $N$ is chosen sufficiently large. It is only necessary to verify that the functions $g_{n}(z)$ have Property $\mathrm{T}$ in some circle $|z|<s_{1}$. An application of Theorem 3.1 shows at once that this is true, with (for example) $s_{1}$ such that

$$
\sup _{0 \leqq n<\infty} H_{n}\left(s_{1}\right)<1 \text {. }
$$

Alternatively, we may suppose that, for $n=0,1,2, \cdots$,

$$
\left|h_{n}(z)\right| \leqq M_{n}(\rho) \quad(|z| \leqq \rho),
$$

and that

$$
M(\rho)=\limsup _{n \rightarrow \infty} M_{n}(r)
$$

is finite. Then we can state

(12) For the theorem under (4.7), see G. S. Ketchum [4]. 
THEOREM 4.2(13). The conclusion of Theorem 3.2 holds if the quantity $M(\rho)$ in (3.9) is defined by (4.8).

Expansions in terms of functions $g_{n}(z)$, analytic in $|z|<r_{0}$, are particularly interesting if the expansion of every $f(z)$ which is analytic in $|z|<s$ converges in every circle $|z| \leqq s^{\prime}<s$ (where naturally $s \leqq r_{0}$ ). This property (which we may call Property $U$ ) is possessed, of course, by the functions $z^{n}$. Using the theorems of this section, we can easily obtain the following sufficient conditions for a set $g_{n}(z)$ to have Property $\mathrm{U}$ :

$$
\begin{aligned}
h\left(r_{0}\right) & <1, \\
L_{r_{0}} & =0, \\
M\left(r_{0}\right) & =0,
\end{aligned}
$$

where $h\left(r_{0}\right), L_{r_{0}}$, and $M\left(r_{0}\right)$ denote the limits of the respective functions of $r$ (defined in (4.4) and (4.8)) as $r \rightarrow r_{0}$. Condition (4.11) shows, for example, that if

$$
h_{n}(z)=o(1), \quad n \rightarrow \infty,
$$

uniformly with respect to $z$ in each circle $|z| \leqq r^{\prime}<r_{0}$, then the set $\left\{g_{n}(z)\right\}$ $=\left\{z^{n}\left[1+h_{n}(z)\right]\right\}$ has Property U. This result was obtained by Sheffer and by Takahashi $\left({ }^{14}\right)$; it generalizes a result of Widder [20], in which the condition $h_{n}(z)=O(1 / n)$ appears instead of (4.12). Condition (4.9) will sometimes establish Property $U$ when (4.12) is not satisfied. For example, if

$$
h_{n}(z)=\sum_{k=1}^{\infty} \delta_{k}^{(n)} z^{k}
$$

and

$$
\left|\delta_{k}^{(n)}\right| \leqq \frac{1}{k(k+1)} \quad(n=0,1, \cdots ; k=1,2, \cdots)
$$

we have

$$
h(z)=1-\frac{1-z}{z} \log (1-z)=\sum_{k=1}^{\infty} \frac{z^{k}}{k(k+1)},
$$

and $h(r)<1$ if $r<1$. In this case the corresponding functions $g_{n}(z)$ have Property $U$ in $|z|<1$, although neither (4.10), (4.11), nor (4.12) is necessarily satisfied.

5. Further generalizations. In Theorems 4.1 and 4.2 we made restrictions on the individual coefficients in the power series of the functions $h_{n}(z)$. In this section a method will be developed for replacing such restrictions by re-

(13) Takenaka [15], G. S. Ketchum [4].

(14) Sheffer [10, pp. 588, 597], Takahashi [13]. Cf. G. S. Ketchum [4, p. 215]. 
strictions on linear combinations of the coefficients. The results obtained (which could evidently be generalized still further) overlap those of G. S. Ketchum [4], who first obtained such results.

Suppose that $L$ is a one-to-one linear operation from $H_{2}(r)$ to $H_{2}(r)$ (so that $L$ has a linear inverse). If the set of functions $G_{n}(z)=L\left[g_{n}(z)\right]$ is a base, and $f(z)$ is an arbitrary element of $H_{2}(r)$, we have a unique expansion

$$
L[f(z)]=\sum_{n=0}^{\infty} a_{n} G_{n}(z) ;
$$

since $L^{-1}$ is continuous, we have

$$
f(z)=\sum_{n=0}^{\infty} a_{n} g_{n}(z)
$$

if we also have

$$
f(z)=\sum_{n=0}^{\infty} b_{n} g_{n}(z)
$$

then

$$
L[f(z)]=\sum_{n=0}^{\infty} b_{n} G_{n}(z),
$$

and $a_{n}=b_{n}(n=0,1,2, \cdots)$. The convergence is convergence in the topology of $H_{2}(r)$; this, as we have seen, implies uniform convergence in every circle $|z| \leqq r^{\prime}<r$. Hence an expansion theorem for the functions $L\left[g_{n}(z)\right]$ gives rise to an expansion theorem for the $g_{n}(z)$ themselves. A trivial, but not unimportant, illustration is given by the operator $L$ which transforms $g_{n}(z)$ into $\sigma(z) g_{n}(z)$, where $\sigma(z)$ is analytic and bounded in $|z| \leqq r, \sigma(0)=1$, and $\sigma(z) \neq 0$ in $|z| \leqq r$.

We now discuss a case which is not entirely covered by the procedure just outlined; it includes some of the results of G. S. Ketchum mentioned above. For simplicity we consider only the special case when the coefficients of the functions $h_{n}(z)$ are combined two at a time. Let $\left\{k_{\nu}\right\}(\nu=1,2, \cdots)$ be a sequence of complex numbers such that

$$
\limsup _{\nu \rightarrow \infty}\left|k_{\nu}\right|^{1 / \nu} \leqq 1
$$

so that $\Lambda(z)=\sum_{1}^{\infty} k_{\nu} z^{\nu}$ is analytic in $|z|<1$. If

we define

$$
f(z)=\sum_{\nu=0}^{\infty} b_{\nu} z^{\nu}, \quad|z|<r,
$$

$$
F(z)=L[f]=f(z)+\sum_{\nu=0}^{\infty} k_{\nu} b_{\nu-1} z^{\nu}=\sum_{\nu=0}^{\infty}\left(b_{\nu}+k_{\nu} b_{\nu-1}\right) z^{\nu} . \quad\left(b_{-1}=0\right)
$$


By Hadamard's multiplication theorem $\left({ }^{15}\right) ; F(z)$ has no singularities inside the circle $|z|<r$. We have the representation

$$
F(z)=f(z)-\frac{1}{2 \pi i} \int_{C} \Lambda(z / w) f(w) d w \quad\left(|z|<r^{\prime}<r\right)
$$

where $C$ is the circle $|w|=r^{\prime}<r$.

Let us now consider the expansion of a given analytic function $f(z)$ in terms of a set of functions

$$
g_{n}(z)=z^{n}\left[1+h_{n}(z)\right],
$$

with $h_{n}(z)$ analytic in $|z|<r$ and $h_{n}(0)=0$. We introduce the functions

$$
F(z)=L[f], \quad G_{n}(z)=L\left[g_{n}\right]
$$

it is clear that we have $G_{n}(z)=z^{n}\left[1+H_{n}(z)\right]$, where the $H_{n}(z)$ are analytic in $|z|<r$ and $H_{n}(0)=0$. (It is not in general true that $G_{n}(z) \varepsilon H_{2}(r)$ if $\left.g_{n}(z) \varepsilon H_{2}(r)\right)$. Let us suppose that the $G_{n}(z)$ satisfy one of the conditions of Theorems 4.1 and 4.2 , so that we have in $|z|<r$ a unique expansion

$$
F(z)=\sum_{n=0}^{\infty} c_{n} G_{n}(z)
$$

converging uniformly in any circle $|z| \leqq s<r$, with

$$
\sum_{n=0}^{\infty}\left|c_{n}\right|^{2} s^{2 n} \leqq A(s) \int_{0}^{2 \pi}\left|F\left(s e^{i \theta}\right)\right|^{2} d \theta
$$

the last relation follows from the remark made at the end of $\S 3$. From (5.2) and (5.3) we then have

$$
f(z)-\frac{1}{2 \pi i} \int_{C} \Lambda(z / w) f(w) d w=\sum_{n=0}^{\infty} c_{n}\left\{g_{n}(z)-\frac{1}{2 \pi i} \int_{C} \Lambda(z / w) g_{n}(w) d w\right\},
$$

the series converging in $|z|<r$, uniformly in $|z| \leqq s<r$. If $s$ is temporarily fixed, and we take $\rho$ so that $s<\rho<r$, the series $\sum\left|c_{n}\right|{ }^{2} \rho^{2 n}$ is convergent, and the functions $w^{-n} g_{n}(w)$ are uniformly bounded on $|w|=s$. It follows, by an application of Cauchy's inequality, that the series $\sum c_{n} g_{n}(w)$ is uniformly (and absolutely) convergent on $.|w|=s$ and so in $|w| \leqq s$. Since the series on the right of (5.4) is uniformly convergent in any circle $|z| \leqq s<r$, we have

$$
f(z)-\sum_{n=0}^{\infty} c_{n} g_{n}(z)-\frac{1}{2 \pi i} \int_{C} \Lambda(z / w)\left\{f(w)-\sum_{n=0}^{\infty} c_{n} g_{n}(w)\right\} d w \equiv 0
$$

in $|z|<r$, both series being uniformly convergent in any circle $|z| \leqq s<r$. That is, the function $f^{*}(z)$ defined by

(15) See, e.g., Dienes [24, p. 346]. 


$$
f^{*}(z)=f(z)-\sum_{n=0}^{\infty} c_{n} g_{n}(z)=\sum_{v=0}^{\infty} b_{\nu}^{*} z^{\nu} \quad\left(b_{-1}^{*}=0\right)
$$

is analytic in $|z|<r$, and we have $L\left[f^{*}(z)\right] \equiv 0$ in $|z|<r$. From (5.1) we see that this means that

$$
b_{\nu}^{*}+k_{\nu} b_{\nu-1}^{*}=0 \quad(\nu=0,1,2, \cdots),
$$

and hence that $b_{\nu}^{*}=0(\nu=0,1,2, \cdots)$. That is,

$$
f(z)=\sum_{n=0}^{\infty} c_{n} g_{n}(z)
$$

the series converging uniformly in any circle $|z| \leqq s<r$. We sum up our conclusions in a formal theorem.

TheOREM 5.1. If the functions $G_{n}(z)=L\left[g_{n}(z)\right]$, where $L$ is defined by (5.1), satisfy the conditions of Theorem 4.1 or Theorem 4.2, and $s$ is defined as in those theorems, the functions $g_{n}(z)$ have Property $\mathrm{T}$ in $|z|<s$.

For example, if the numbers $k_{\nu}$ satisfy

$$
\limsup _{\nu \rightarrow \infty}\left|k_{\nu}\right|^{1 / \nu} \leqq 1
$$

if

$$
\begin{array}{cr}
h_{n}(z)=\sum_{\nu=1}^{\infty} \gamma_{\nu}^{(n)} z^{\nu} & \left(|z|<r_{0}\right), \\
\left|\gamma_{\nu}^{(n)}+k_{n+\nu} \gamma_{\nu-1}^{(n)}\right| \leqq \beta_{\nu} & (n=0,1, \cdots ; \nu=1,2, \cdots), \\
h(z)=\sum_{\nu=1}^{\infty} \beta_{\nu} z^{\nu} & \left(|z|<r_{0}\right),
\end{array}
$$

and $h(s)<1$, then the functions $z^{n}\left[1+h_{n}(z)\right]$ have Property $\mathrm{T}$ in $|z|<s$.

It is clear that linear operations other than that defined in (5.1) could also be used.

6. Special expansion theorems.

TheOREM 6.1. If $\phi(z)$ is an analytic function whose Maclaurin series has positive coefficients $\left({ }^{16}\right)$, and radius of convergence $R(R \leqq \infty)$, if $\phi(0)=1$, and if the complex numbers $\alpha_{n}$ satisfy

$$
\limsup _{n \rightarrow \infty}\left|\alpha_{n}\right| \leqq 1, \quad \sup _{0 \leqq n<\infty}\left|\alpha_{n}\right|<R / \phi^{-1}(2),
$$

the functions

$$
z^{n} \phi\left(\alpha_{n} z\right) \quad(n=0,1,2, \cdots)
$$

have Property $\mathrm{T}$ in any circle $|z| \leqq s<\phi^{-1}(2)$.

(16) That is, $\phi(z)$ is absolutely monotonic on the segment $(0, R)$ of the real axis. 
Here we have $h_{n}(z)=\phi\left(\alpha_{n} z\right)-1$; and, in the notation of $\S 4$,

$$
H_{n}(r)=\phi\left(\left|\alpha_{n}\right| r\right)-1, \quad h(r) \leqq \phi(r)-1,
$$

and $h(r)<1$ if $\phi(r)<2$.

In particular, we may have $\left({ }^{17}\right) \phi(z)=e^{z}$.

Various modifications of the situation considered in Theorem 6.1 are possible. We shall discuss three which have interesting applications.

THEOREM 6.2. The functions $g_{n}(z)$ defined by

$$
\begin{aligned}
& g_{0}(z)=1, \\
& g_{n}(z)=z^{n-1} \frac{e^{\alpha_{n} z}-e^{\beta_{n} z}}{\alpha_{n}-\beta_{n}} \\
& \quad\left(\left|\alpha_{n}\right| \leqq 1,\left|\beta_{n}\right| \leqq 1, \alpha_{n} \neq \beta_{n} ; n=1,2, \cdots\right)
\end{aligned}
$$

have Property $\mathrm{T}$ in the circle $|z|<r$ if $r<\log 2$.

It is easy to show that we may take $h(r)=e^{r}-1$ in this case. For details, the reader is referred to the author's note [22] where Theorem 6.2 is applied to show that an entire function of exponential type less than $\log 2$ has an infinite number of derivatives which are univalent in the unit circle, unless it is a polynomial.

For the next two theorems, it is necessary to go back to Theorem 2.2.

ThEOREM 6.3. The functions $g_{n}(z)$ defined by

$$
\begin{aligned}
g_{2 n}(z) & =z^{2 n} e^{\alpha_{n} z} & (|\alpha| \leqq 1), \\
g_{2 n+1}(z) & =z^{2 n+1}, &
\end{aligned}
$$

have Property $\mathrm{T}$ in any circle $|z|<r<0.780$.

We note that $\log 2=0.693, \pi / 4=0.785$. We thus have more than Theorem 6.1 would establish, but still less than the result which may be conjectured $\left({ }^{18}\right)$, that Theorem 6.1 holds, when $\phi(z)=e^{z}$, for $s<\pi / 4$.

By Theorem 2.2, Theorem 6.3 will follow if we show that for every sequence $\left\{a_{n}\right\}$ of complex numbers and for every $N$

$$
\frac{1}{2 \pi} \int_{0}^{2 \pi}\left|\sum_{n=0}^{N} a_{n}\left[g_{n}\left(r e^{i \theta}\right)-r^{n} e^{i n \theta}\right]\right|^{2} d \theta \leqq \lambda(r) \sum_{n=0}^{N}\left|a_{n}\right|^{2} r^{2 n}, \quad r<0.780,
$$

with $\lambda(r)<1$. The left-hand side, by the reasoning of Theorem 3.1 , does not exceed

(17) The corresponding theorem, with region of convergence $|z|<1 / e$, was given by Takenaka [15]. See also footnote 2.

(18) See footnote 21 . 


$$
\begin{aligned}
\frac{1}{2 \pi} \int_{0}^{2 \pi} & \left|e^{z}-1\right|^{2}\left|\sum_{n=0}^{[N / 2]} a_{2 n} z^{2 n}\right|^{2} d \theta \quad\left(z=r e^{i \theta}\right) \\
& =\frac{1}{2 \pi} \int_{0}^{2 \pi}\left|e^{z}-1\right|^{2}|\psi(z)|^{2} d \theta \\
& =\frac{1}{2 \pi} \int_{-\pi / 2}^{\pi / 2}\left|\psi(z)\left(e^{z}-1\right)\right|^{2} d \theta+\frac{1}{2 \pi} \int_{-\pi / 2}^{\pi / 2}\left|\psi(z)\left(e^{-z}-1\right)\right|^{2} d \theta,
\end{aligned}
$$

since $|\psi(z)|$ is periodic in $\theta$ with period $\pi$. Thus the left side of (6.3) does not exceed

$$
\begin{aligned}
& \frac{1}{2 \pi}\left\{\left(e^{r}-1\right)^{2}+\max _{-\pi / 2 \leqq \theta \leqq \pi / 2}\left|e^{-z}-1\right|^{2}\right\} \int_{-\pi / 2}^{\pi / 2}|\psi(z)|^{2} d \theta \\
& \quad=\frac{1}{2}\left\{\left(e^{r}-1\right)^{2}+\max _{-\pi / 2 \leqq \theta \leqq \pi / 2}\left|e^{-z}-1\right|^{2}\right\} \sum_{n=0}^{[N / 2]}\left|a_{2 n}\right|^{2} r^{4 n}
\end{aligned}
$$

We have

$$
\begin{aligned}
\left|e^{-z}-1\right|^{2} & =e^{-2 r \cos \theta}-2 e^{-r \cos \theta} \cos (r \sin \theta)+1 \\
& \leqq e^{-2 r \cos \theta}+1-2 e^{-r \cos \theta} \cos r \\
& =A(\theta),
\end{aligned}
$$

say. Now

$$
A^{\prime}(\theta)=2 r \sin \theta e^{-r \cos \theta}\left(e^{-r \cos \theta}-\cos r\right),
$$

and vanishes only when $\theta=0$ or when $\cos \theta=(1 / r) \log \cos (1 / r)$. In the latter case, $\exp (-r \cos \theta)=\cos r$, and

$$
A(\theta)=1-\cos ^{2} r<2(1-\cos r)=A\left(\frac{1}{2} \pi\right) .
$$

Consequently $A(\theta)$ assumes its maximum when $\theta=0$ or $\theta=\frac{1}{2} \pi$. For $r=0.780$, we find that $A(0)<A\left(\frac{1}{2} \pi\right)$. In fact, this inequality is

$$
e^{-2 r}-2 e^{-r}+1<2(1-\cos r)+2 e^{-r} \cos r-2 e^{-r} \text {, }
$$

which is equivalent to

$$
1+e^{-r}>2 \cos r
$$

which is satisfied for $r=0.780$. Hence

$$
A(\theta) \leqq A\left(\frac{1}{2} \pi\right)=2(1-\cos r),
$$

and the left side of (6.3) does not exceed

$$
\frac{1}{2}\left\{\left(e^{r}-1\right)^{2}+2(1-\cos r)\right\} \sum_{n=0}^{N}\left|a_{n}\right|^{2} r^{2 n} ;
$$

the brace is less than 2 when $r=0.780$. This completes the proof of Theorem 6.3. 
THEOREM 6.4. If $r<\log 2$, and the complex numbers $\beta_{n}$ are such that

$$
\sum_{n=0}^{\infty}\left|\beta_{n}\right|{ }^{2} r^{-2 n}<2 e^{r}-e^{2 r}
$$

the functions $g_{n}(z)$ defined by

$$
g_{n}(z)=z^{n} e^{\alpha_{n} z}-\beta_{n}
$$$$
\left(\left|\alpha_{n}\right| \leqq 1\right)
$$

have Property $\mathrm{T}$ in $|z|<r$.

To apply Theorem 2.2, we need to show that if $h_{n}(z)=e^{\alpha_{n} z}-1$, then for all $\left\{a_{n}\right\}$ and $N$,

$$
\frac{1}{2 \pi} \int_{0}^{2 \pi}\left|\sum_{n=0}^{N} a_{n}\left[z^{n} h_{n}(z)-\beta_{n}\right]\right|^{2} d \theta \leqq \lambda(r) \sum_{n=0}^{N}\left|a_{n}\right|^{2} r^{2 n} \quad\left(z=r e^{i \theta}\right),
$$

with $\lambda(r)<1$, when $r$ and $\left\{\beta_{n}\right\}$ satisfy (6.4).

The left side of (6.6) may be written in the form

$$
\begin{aligned}
\frac{1}{2 \pi} \int_{0}^{2 \pi} & \left|\sum_{n=0}^{N} a_{n} z^{n} h_{n}(z)-\sum_{n=0}^{N} a_{n} \beta_{n}\right|^{2} d \theta \\
= & \frac{1}{2 \pi} \int_{0}^{2 \pi}\left|\sum_{n=0}^{N} a_{n} z^{n} h_{n}(z)\right|^{2} d \theta-\frac{1}{\pi} \int_{0}^{2 \pi} \Re\left\{\sum_{n=0}^{N} a_{n} z^{n} h_{n}(z) \sum_{m=0}^{N} \bar{a}_{m} \bar{\beta}_{m}\right\} d \theta \\
& \quad+\left|\sum_{n=0}^{N} a_{n} \beta_{n}\right|^{2} \\
= & S_{1}-S_{2}+S_{3} .
\end{aligned}
$$

Now, by the proof of Theorem 3.1,

$$
S_{1} \leqq\left(e^{r}-1\right)^{2} \sum_{n=0}^{N}\left|a_{n}\right|^{2} r^{2 n}
$$

and

$$
S_{8} \leqq\left(\sum_{n=0}^{N}\left|a_{n}\right| r^{n}\left|\beta_{n}\right| r^{-n}\right)^{2} \leqq \sum_{n=0}^{N}\left|a_{n}\right|{ }^{2} r^{2 n} \sum_{n=0}^{N}\left|\beta_{n}\right|^{2} r^{-2 n}
$$

by Cauchy's inequality. Finally,

$$
S_{2}=2 \Re\left\{\frac{1}{2 \pi} \int_{0}^{2 \pi} \sum_{n=0}^{N} a_{n} z^{n} h_{n}(z) \sum_{m=0}^{N} \bar{a}_{m} \bar{\beta}_{m} \frac{d z}{i z}\right\}=0,
$$

since $h_{n}(0)=0$. Combining this with (6.7) and (6.8), we have

$$
\frac{1}{2 \pi} \int_{0}^{2 \pi}\left|\sum_{n=0}^{N} a_{n}\left[z^{n} h_{n}(z)-\beta_{n}\right]\right|^{2} d \theta \leqq\left\{\left(e^{r}-1\right)^{2}+\sum_{n=0}^{N}\left|\beta_{n}\right|{ }^{2} r^{-2 n}\right\} \sum_{n=0}^{N}\left|a_{n}\right|^{2} r^{2 n}
$$


Thus by Theorem 2.2 the system (6.5) has Property $\mathrm{T}$ if the brace in the last inequality is less than one. This will clearly be true if (6.4) is satisfied.

7. Applications. We now use the theorems of $\$ 6$ to prove theorems concerning the values taken by derivatives of entire functions of order one and exponential type. We need the following lemma.

Lemma $\left({ }^{19}\right)$. If $f(z)$ is an entire function of exponential type $k$, it has the representation

$$
f(z)=\int_{C} e^{z w} F(w) d w,
$$

where $C$ is any circle $|z|=k^{\prime}>k$, and $F(w)$ is analytic outside $|z|=k$.

It follows that

$$
f^{(n)}(z)=\int_{C} w^{n} e^{z w} F(w) d w \quad(n=1,2, \cdots) .
$$

If now the functions $g_{n}(w)$ have property $\mathrm{T}$ in $|w| \leqq k^{\prime}$, we can expand the function $e^{z w}$ in terms of them, substitute in (7.1), and integrate term by term $\left({ }^{20}\right)$. We thus obtain an expansion of the form

$$
f(z)=\sum_{n=0}^{\infty} c_{n}(z) \int_{C} g_{n}(w) F(w) d w .
$$

We can now establish the following theorems.

THEOREM 7.1. If $f(z)$ is an entire function of exponential type $k<\log 2$, and if $f(0)=1,\left|\alpha_{n}\right| \leqq 1$, and $r<k$, the inequality

$$
\sum_{n=0}^{\infty}\left|f^{(n)}\left(\alpha_{n}\right)\right|^{2} r^{-2 n} \geqq 2 e^{r}-e^{2 r}
$$

is valid.

If (7.4) is not true, Theorem 6.4 applies, with $\beta_{n}=f^{(n)}\left(\alpha_{n}\right)$, and (7.3) has the form

$$
f(z)=\sum_{n=0}^{\infty} c_{n}(z)\left\{f^{(n)}\left(\alpha_{n}\right)-\beta_{n} f(0)\right\}=0,
$$

which is impossible since $f(0)=1$.

As a corollary we obtain the following theorem of S. Takenaka [29].

THEOREM 7.2. If $f(z)$ is an entire function of exponential type $k<\log 2$, $\operatorname{and}\left({ }^{21}\right)\left|\alpha_{n}\right| \leqq 1$, then

(19) See Polya [27, pp. $580 \mathrm{ff}$.].

(20) Cf. Whittaker [30, p. 67], Gelfond [25].

(21) Or even if $\lim \sup \left|\alpha_{n}\right| \leqq 1$. 
implies $f(z) \equiv 0$.

For, if $f(0) \neq 0$, we consider $f(z) / f(0)$, to which Theorem 7.1 applies, since the left side of (7.4) is zero if (7.5) is satisfied. If $f(0)=0$, while $f\left(z_{0}\right) \neq 0$ for some $z_{0}$ in $|z|<1$, we apply Theorem 7.1 to the function $\left[f\left(z_{0}\right)-f(z)\right] / f\left(z_{0}\right)$, taking $\alpha_{0}=z_{0}$.

From Theorem 6.3, we obtain

TheOREM 7.3. If $f(z)$ is an entire function of exponential type $k<0.780$, and $\left|\alpha_{n}\right| \leqq 1$, then the conditions

$$
f^{(2 n+1)}(0)=f^{(2 n)}\left(\alpha_{n}\right)=0 \quad(n=0,1,2, \cdots)
$$

imply that $f(z) \equiv 0$.

This is more than follows from Theorem 7.2, but less than would follow if Theorem 7.2 were proved to be true with $k<\pi / 4$, as has been conjectured $\left({ }^{22}\right)$.

Analogous theorems concerning functions analytic in a finite $\operatorname{circle}\left({ }^{23}\right)$ can be proved by developing $(w-z)^{-1}$, as a function of $w$, in terms of (for example)

$$
\frac{w^{n}}{\left(1-\alpha_{n} w\right)^{n}},
$$

and substituting the expansion into Cauchy's integral formula.

PAPERS ON GENERAL EXPANSION TheOREMS

1. G. D. Birkhoff, Sur une généralisation de la série de Taylor, Comptes Rendus Hebdomadaires des Séances de l'Académie des Sciences, Paris, vol. 163 (1917), pp. 942-945.

2. R. F. Graesser, A certain general type of Neumann expansions and expansions in confluent hypergeometric functions, American Journal of Mathematics, vol. 49 (1927), pp. 577-597.

3. S. Izumi, On the expansion of analytic function, Tôhoku Mathematical Journal, vol. 28 (1927), pp. 97-106.

4. G. S. Ketchum, On certain generalizations of the Cauchy-Taylor expansion theory, these Transactions, vol. 40 (1936), pp. 208-224.

5. P. W. Ketchum, Infinite systems of linear equations and expansions of analytic functions, Duke Mathematical Journal, vol. 4 (1938), pp. 668-677.

6. T. Kubota, Eine Verallgemeinerung des Taylor-Cauchyschen Satzes, Tôhoku Mathematical Journal, vol. 22 (1923), pp. 336-347.

7. S. Narumi, $A$ theorem on the expansion of analytic functions, Tóhoku Mathematical Journal, vol. 30 (1929), pp. 441-444.

8. Y. Okada, On a certain expansion of analytic function, Tôhoku Mathematical Journal, vol. 22 (1923), pp. 325-335.

9. S. Pincherle, Sopra alcuni sviluppi in serie per funzioni analitiche, Memorie della Reale Accademia delle Scienze dell'Istituto di Bologna, (4), vol. 3 (1881), pp. 151-180.

(22) See Whittaker [30, p. 45], Schoenberg [28].

(28) See Takenaka $[15,29]$; Whittaker $[30$, p. 43$]$. 
10. I. M. Sheffer, Concerning some methods of best approximation, and a theorem of Birkhoff, American Journal of Mathematics, vol. 57 (1935), pp. 587-614.

11. S. Takahashi, On the expansion of analytic function, Proceedings of the Imperial Academy, Tokyo, vol. 6 (1930), pp. 389-392.

12. - A remark on Mr. D. V. Widder's theorem, Tôhoku Mathematical Journal, vol. 33 (1930), pp. 48-54.

13. - On the expansion of analytic functions, Tôhoku Mathematical Journal, vol. 35 (1932), pp. 242-243.

14. S. Takenaka, A generalization of Taylor's series, Japanese Journal of Mathematics, vol. 7 (1930), pp. 187-198.

15. - On the expansion of analytic functions in series of analytic functions and its application to the study of the distribution of zero points of the derivatives of analytic functions, Nippon Sugaku-Buturigakkwai Kizi (Proceedings of the Physico-Mathematical Society of Japan), (3), vol. 13 (1931), pp. 111-132.

16. L. Tonelli, Sulle serie di funzioni analitiche della forma $\sum a_{n}(x) x^{n}$, Annali di Matematica Pura ed Applicata, (3), vol. 18 (1911), pp. 99-103.

17. J. L. Walsh, On the expansion of analytic functions in series of polynomials, these Transactions, vol. 26 (1924), pp. 155-170.

18. - On the expansion of analytic functions in series of polynomials and in series of other analytic functions, these Transactions, vol. 30 (1928), pp. 307-332.

19. - Note on the expansion of analytic functions in series of polynomials and in series of other analytic functions, these Transactions, vol. 31 (1929), pp. 53-57.

20. D. V. Widder, On the expansion of analytic functions of a complex variable in generalized Taylor's series, these Transactions, vol. 31 (1929), pp. 43-52.

\section{OTHER REFERENCES}

21. S. Banach, Théorie des Opérations Linéaires, 1932.

22. R. P. Boas, Jr., Univalent derivatives of entire functions, Duke Mathematical Journal, vol. 6 (1940), pp. 719-721.

23. H. F. Bohnenblust and A. Sobczyk, Extensions of functionals on complex linear spaces, Bulletin of the American Mathematical Society, vol. 44 (1938), pp. 91-93.

24. P. Dienes, The Taylor Series, An Introduction to the Theory of Functions of a Complex Variable, 1931.

25. A. Gelfond, Interpolation et unicité des fonctions entières, Matematicheskii Sbornik (Recueil Mathématique), new series, vol. 4 (1938), pp. 115-147.

26. R. E. A. C. Paley and N. Wiener, Fourier Transforms in the Complex Domain, American Mathematical Society Colloquium Publications, vol. 19, 1934.

27. G. Pólya, Untersuchungen über Lücken und Singularitäten von Potenzreihen, Mathematische Zeitschrift, vol. 29 (1929), pp. 549-640.

28. I. J. Schoenberg, On the zeros of successive derivatives of integral functions, these Transactions, vol. 40 (1936), pp. 12-23.

29. S. Takenaka, On the expansion of integral transcendental functions in generalized Taylor's series, Nippon Sûgaku-Buturigakkwai Kizi (Proceedings of the Physico-Mathematical Society of Japan), (3), vol. 14 (1932), pp. 529-542.

30. J. M. Whittaker, Interpolatory Function Theory, 1935.

31. A. Zygmund, Trigonometrical Series, 1935.

32. I. I. Ibragimoff (I. Ibraguimoff), Sur quelques systèmes complets de fonctions analytiques (in Russian), Izvestiya Akademii Nauk SSSR, Seriya Matematicheskaya (Bulletin de l'Académie des Sciences de l'URSS, Série Mathématique), 1939, pp. 553-567; French summary, pp. 567-568.

\section{Duke UNIVERSITY,}

Durham, N. C. 